\title{
Systematic numerical study of spin-charge separation in one dimension
}

\author{
M. G. Zacher, E. Arrigoni and W. Hanke \\ Institut für Theoretische Physik, Universität Würzburg, D-97074 Würzburg, Germany \\ J. R. Schrieffer \\ NHMFL and Department of Physics, Florida State University, Tallahassee, Florida 32310
}

\begin{abstract}
The problem of spin-charge separation is analyzed numerically in the metallic phase of the one-band Hubbard model in one dimension by studying the behavior of the single-particle Green's function and of the spin and charge susceptibilities. We first analyze the Quantum-Monte Carlo data for the imaginary-time Green's function within the Maximum Entropy method in order to obtain the spectral function at real frequencies. For some values of the momentum sufficiently away from the Fermi surface two separate peaks are found, which can be identified as charge and spin excitations. In order to improve our accuracy and to be able to extend our study to a larger portion of the Brillouin zone, we also fit our data with the imaginary-time Green's function obtained from the Luttinger-model solution with two different velocities as fitting parameters. The excitation energies associated with these velocities turn out to agree, in a broad range of momenta, with the ones calculated from the charge and spin susceptibilities. This allows us to identify these single-particle excitations as due to a separation of spin and charge. Remarkably, the range of momenta where spin-charge separation is seen extends well beyond the region of linear dispersion about the Fermi surface. We finally discuss a possible extension of our method to detect spin-charge separation numerically in two dimensions.
\end{abstract}

PACS numbers : 71.10.Pm, 71.15.-m, 71.10.Fd, 71.10.Hf

\section{INTRODUCTION}

One-dimensional (1D) interacting fermion systems show a number of anomalous properties which cannot be understood in the framework of the Fermi-liquid theory of normal metals. In particular, their momentum distribution and density of states are in sharp contrast with Fermi-liquid theory for energies and momenta close to the Fermi surface. In general, 1D systems can be described by an effective low-energy theory based on the exactly solvable Luttinger model with suitably renormalized parameters and are thus referred to as Luttinger liquids (LL). 3 One of the most striking features of the Luttinger model is the complete separation of spin and charge degrees of freedom which manifests itself in the splitting of the single-particle spectral function in two peaks corresponding to spin and charge excitations propagating independently 6.6 Another important characteristic of the Luttinger model is the presence of powerlaw behavior with interaction-dependent exponents for various correlation functions.

Beside its application to 1-D systems, LL theory has received particular attention in the past years in the framework of the theory of high- $\mathrm{T}_{c}$ superconductors. The normal phase of the high- $\mathrm{T}_{c} \mathrm{CuO}_{2}$ planes shows in fact a number of anomalous properties which can be possibly understood, if one assumes that the $\mathrm{fu}_{2}$ planes are in a kind of two-dimensional LL state 11 In particular, it has been suggested that spin-charge separation could be present also in the $\mathrm{CuO}_{2}$ planes and that it plays an essential role in the-yay particles are allowed to tunnel between the planes. 12
Numerical methods have been proven to be crucial for the theoretical understanding of models describing the $\mathrm{CuO}_{2}$ planes, since electron correlation is rather strong in these systems and perturbative methods are necessarily limited. Spin-charge separation is predicted exactly for the Luttinger model : an ideal exactly-solvable model. It is thus important to test numerically to what extent spin-charge separation can occur in a one-dimensional physical model. Moreover, in order to prove the theories mentioned above, it would be important to check whether some two-dimensional models exist, which display spin-charge separation. Recently, there have been several attempts to detect spin-charge separation in onebut also in two-dimensional models. In the $U=\infty 1$ D Hubbard mode $\mathbb{1}^{13}$ spin-charge separation occurs in a natural way at all energies (and not only at low energies like expected in a $L L$ ) due to the exact factorization of the wave function.14 In a numerical work Jagla et al.15 have observed the propagation in real time of a singleelectron wave packet created at a time $t=0$ in a $1-\mathrm{D}$ Hubbard model. This wave packet splits up in two excitations propagating with different velocities that can be associated with charge and spin. In a work by W. O. Puttika and collaborators 16 the possibility of spin-charge separation in the 2D $t-J$ model has been signaled by the presence of two distinct characteristic wave vectors for the spin and charge degrees of freedom. Exact diagonalization of the 1-D $t-J$ model 17 have evidenced the presence of two peaks in the single-particle spectral function whose positions scale with $t$ and $J$, respectively, and have thus been identified with charge and spin excitations. In another study 18 two peaks have been de- 
tected in the single-particle spectral function of a $1 \mathrm{D}$ $t-J$ model with corresponding peaks in the charge and spin susceptibilities. These two peaks can be seen, however, only for the momenta which are immediately next to the Fermi momentum and thus they cannot be associated with a dispersive spinon and holon band. Finally, Kim and coworker 19 have detected two dispersive bands for $k<k_{F}$ in a 1-D $t-J$ model close to half filling. This is not surprising, since spin-charge separation is in fact quite natural to expect when one hole is added beyond half filling. The added hole decomposes indeed in a spinless hole and a spin misalignment which propagate with different velocities.19 Nevertheless, the interesting result of that work is that the photoemission spectrum of $\mathrm{SrCuO}_{2}$, also showing two dispersive bands, is remarkably well reproduced by the numerical results.

In this work, we present a systematic QuantumMonte-Carlo study of spin-charge separation away from half filling, where Luttinger-liquid theory is expected to hold, in the whole Brillouin zone (BZ). The nontrivial prediction of Luttinger-liquid theory is, in fact, that spincharge separation occurs in the metallic phase, where the band dispersion is linear. Spin-charge separation at half filling, as studied in the model of Ref. 19, is, in our opinion, of a different nature, since in the insulating phase the holon dispersion is quadratic instead of linear. Of course, in the case of Ref. 19 it was necessary to remain in the insulating phase, since the physical system in study was half-filled.

For some values of the momentum $k$ we are able to see two peaks in the single-particle spectral function which correspond to the spin and charge excitations. However, due to the limited resolution of the Maximum Entropy method, it is not possible to resolve the two peaks in most of the BZ.20 For this reason, in the rest of the BZ we work with the imaginary-time Green's function $\mathcal{G}(k, \tau)$ which is obtained directly from Quantum-Monte-Carlo data without the need of analytic continuation. This has the advantage that one does not need to introduce a further source of error produced by the analytic continuation to real frequencies. Specifically, we perform a nonlinear $\chi^{2}$ fit of $\mathcal{G}(k, \tau)$ by using the solution of the Luttinger model $\mathcal{G}_{v_{1}, v_{2}, K_{\rho}}^{(L M)}(k, \tau)$ with two velocities $v_{1}, v_{2}$, and a normalization constant $c$ as fitting parameters.21 Our fit yields a finite value of the difference $v_{2}-v_{1}$ larger than the statistical error in a large portion of the Brillouin zone. Moreover, the fitted values of the corresponding excitation energies $v_{1}\left(k-k_{F}\right)$ and $v_{2}\left(k-k_{F}\right)$ coincide, within the statistical error, with the spin and charge excitations, respectively, calculated independently via the associated susceptibilities. It is remarkable that this behavior extends well beyond the region of linear dispersion around $k_{F}$ where Luttinger liquid behavior is expected.

Our paper is organized as follows. In Sec. II), we introduce the model, and we show the results of the QuantumMonte-Carlo simulation and analytic continuation to real frequencies by means of the Maximum Entropy method.
In Sec. III, we discuss and show the results of our fit of the imaginary-time Green's function with the result from the Luttinger model. Finally we draw our conclusions in Sec. [V].

\section{QUANTUM-MONTE-CARLO SIMULATION}

We consider the 1D-Hubbard model with periodic boundary conditions described by the following Hamiltonian:

$$
H=-t \sum_{i, \sigma}\left(c_{i+1, \sigma}^{\dagger} c_{i, \sigma}+\text { h.c. }\right)+U \sum_{i} n_{i \downarrow} n_{i \uparrow},
$$

where $c_{i, \sigma}^{(\dagger)}$ are annihilation (creation) operators for an electron at site $i$ with spin $\sigma$ and $n_{i \sigma}=c_{i, \sigma}^{\dagger} c_{i, \sigma}$. The energy scale $t$ of the model will be set to unity in the rest of the paper.

The simulations were carried out with the grandcanonical Quantum-Monte-Carlo method22, 23 on a 64site lattice with inverse temperature $\frac{1}{k_{B} T}=\beta=20$, Hubbard repulsion $U=4$ and an electron density of $\langle n\rangle \approx 0.75$. The simulations yield the one- and twoparticle Green's functions at discrete imaginary times $\tau$ with $0 \leq \tau \leq \beta$. We used a discretization of the imaginary-time axis $\Delta \tau=0.0625$ The spectra (one-particle photoemission spectrum, charge- and spinsusceptibilities) were then obtained by analytically continuing the imaginary-time results to realfrequencies by means of the Maximum-Entropy method24.25.20.

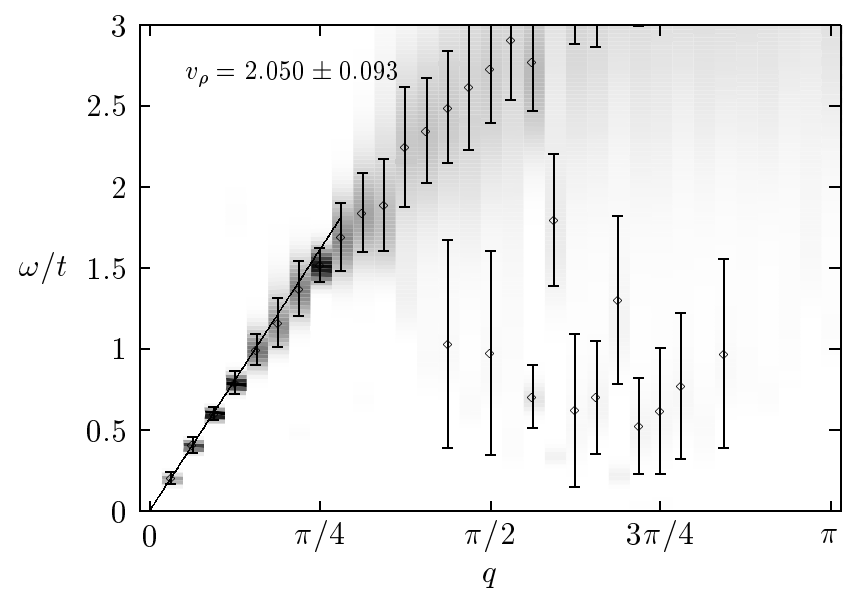

FIG. 1. Density plot of the charge susceptibility $\chi_{\rho}(q, \omega)$ as obtained by the analytic continuation of the Quantum-Monte-Carlo charge-charge correlation function with the Maximum-Entropy method. The grayscale corresponds to the value of $\chi_{\rho}(q, \omega)$ (darker regions correspond to larger values of $\left.\chi_{\rho}(q, \omega)\right)$ and the dots with errorbars show the peak position with their uncertainty. The linear fit (straight line) for small q yields the charge velocity $v_{\rho}$ as indicated in the upper left corner.

Figures 1 and 2 show a density plot of the chargeand spin susceptibilities $\chi_{\rho}$ and $\chi_{\sigma}$, respectively. The 


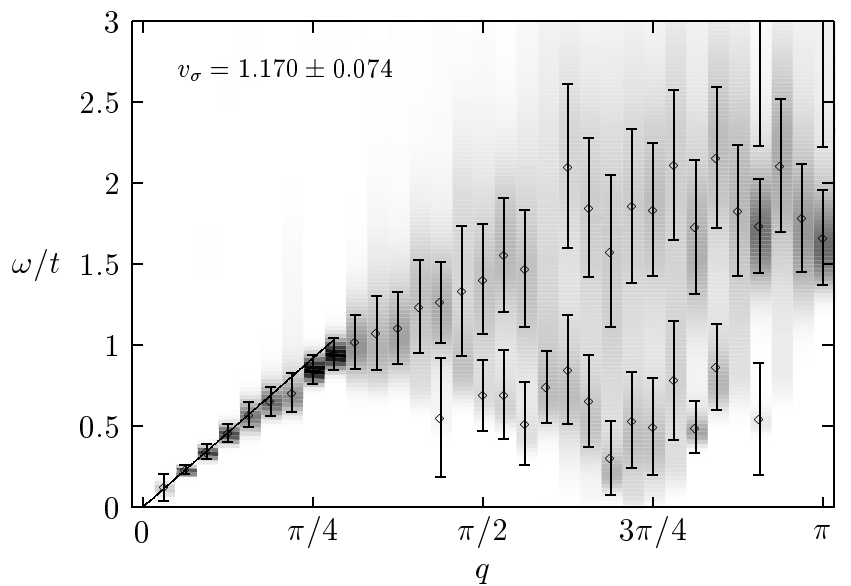

FIG. 2. Density plot of the spin susceptibility $\chi_{\sigma}(q, \omega)$ with the same conventions as Fig. 11 The linear fit (straight line) yields the spin velocity $v_{\sigma}$.

grayscale gives a measure for the value of $\chi_{\rho / \sigma}(q, \omega)$ as a function of momentum transfer $q$ and excitation energy $\omega$. The dispersion relation for spin- and chargeexcitations is defined by the maxima of $\chi_{\rho / \sigma}$ which are indicated by dots with errorbars in the figure. A linear fit of these maxima near $q=0$ yields the spin- and chargevelocities $v_{\sigma}=1.170 \pm 0.074$ and $v_{\rho}=2.050 \pm 0.093$ which agree very well_within the statistical error) with Bethe-Ansatz results26 for the infinite lattice and zero temperature.

However, it is not sufficient to have two different velocities (or, equivalently, energy dispersions) for the twoparticle spin and charge modes in order to conclude that the system shows spin-charge separation. In fact, in a Fermi liquid there are spin and charge excitations that originate from collective modes and do not destroy the quasiparticle 27. The quasiparticles thus remain welldefined and do not split into a charge and a spin excitation as it occurs in a Luttinger liquid. On the other hand, in a Luttinger liquid (or in spin-charge separated system in general) a particle injected at a certain point $x$ decays into a spinon and a holon propagating with different velocities. The separation of the two excitations could then be detected by means of a "diagnostic operator" measuring the time dependence of spin and charge at a given point $y$ far away from $x$. In the case of spin-charge separation, this diagnostic operator would then measure two different passing times for the charge and spin perturbations of the injected particle. True spin-charge separation in the sense of the Luttinger model should be thus identified with different energy dispersions in the spin and charge susceptibilities associated with corresponding low-lying excitations in the single-particle spectrum .18

In Fig. 3 we plot this single-particle spectrum $A(k, \omega) 20$ in the whole Brillouin zone. Close to the Fermi momentum the band dispersion is approximately linear, which justifies the mapping to the Luttinger model. However, the spectrum becomes broader when going away from

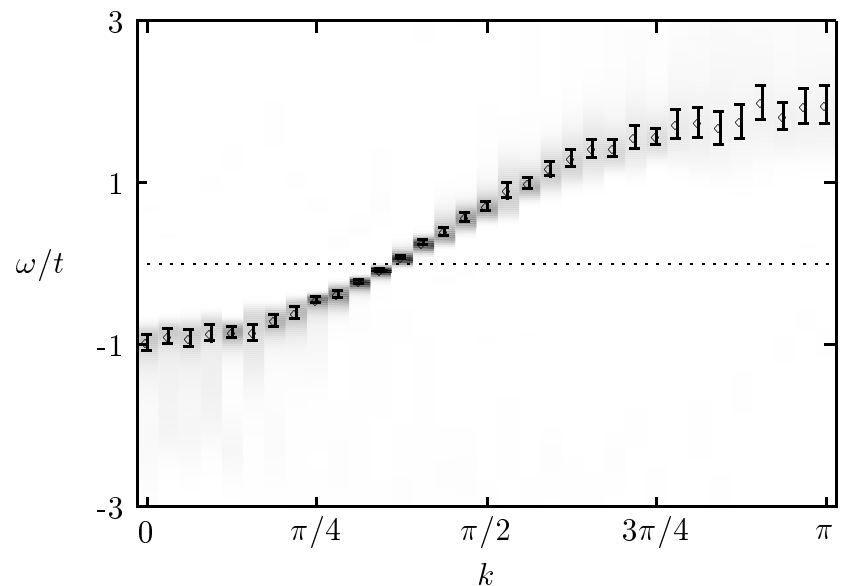

FIG. 3. Density plot of the single-particle photoemission spectrum $A(k, \omega)$ with the same conventions as Fig. 11. It is seen that the dispersion around the Fermi energy (dotted line) is linear over a broad momentum range thus justifying our Luttinger-liquid ansatz for the single-particle Green's function.

the Fermi surface. This phenomenon has two reasons: First, the resolution of the maximum-entropy method gets worse at higher energies, due to the exponential kernel in the spectral theorem, and second, according to Luttinger liquid theory, the single peak starts to split into two peaks representing the spin- and charge-excitations propagating with different velocities. However, for $k$ very close to $k_{F}$ these two peaks, which should be separated by an energy $\left(v_{2}-v_{1}\right)\left(k-k_{F}\right)$, are still too close together for the Maximum Entropy method to distinguish them. On the other hand, at larger values of $\left(k-k_{F}\right)$ the excitation energies are too high and the maximum entropy method becomes less reliable as explained above. In both these cases the two peaks merge into a single broader peak and spin-charge separation is not detectable. There are, however, some favorable intermediate $k$-points where spin-charge separation is directly detectable in the singleparticle spectral function. In figure 1 we show the spectral function for one of these favorable points. Here $k$ is neither too close nor too far from the Fermi surface and the maximum-entropy method (without using any prior knowledge) yields two well-separated peaks. Their positions are consistent with the spin and charge excitation energies (indicated by two dots with horizontal errorbars) calculated independently from the spin- and charge- velocities $\left(\omega_{\rho / \sigma}=\Delta k v_{\rho / \sigma}\right)$. Previously, it was not possible to resglve spin-charge separation in the oneparticle spectrum 20 mainly because they were carried out in a low doping regime ( $\langle n\rangle$ close to 1$)$ where the difference of spin- and charge-velocities is relatively small 26 


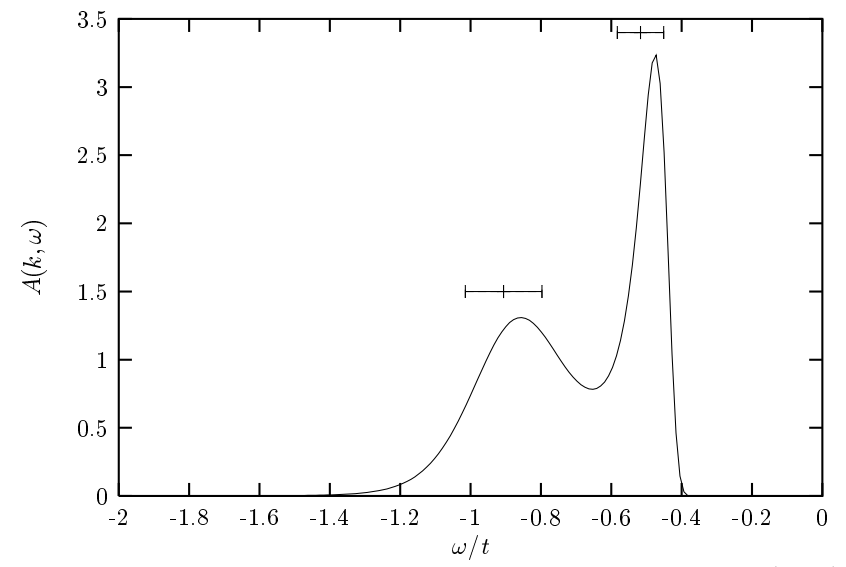

FIG. 4. Single-particle photoemission spectrum $A(k, \omega)$ (in arbitrary units) for $k-k_{F}=-4.5 \frac{\pi}{32}$. The dots with horizontal errorbars indicate the position of spin- and charge-excitations calculated by $\omega_{\rho / \sigma}=\left(k-k_{F}\right) v_{\rho / \sigma}$ with $v_{\rho / \sigma}$ obtained from Figs. 11 and 2. For this $k$-point close to the Fermi momentum the Maximum-Entropy method is able to resolve two separate peaks in the spectral function which can be identified as the spinon and holon excitation, respectively.

\section{FIT OF THE IMAGINARY-TIME GREEN'S FUNCTION}

In order to carry out a systematic study of spin-charge separation it is important to detect spin and charge excitations over the whole BZ, or at least in an extended region around the Fermi surface. However, due to the additional rather large error introduced by the Maximum Entropy analytic continuation method to the QuantumMonte-Carlo data, this turns out to be very difficult for many $k$ points, as we have discussed above. For this reason, we work directly with the data for the imaginarytime Green's function $\mathcal{G}(k, \tau)$. In the asymptotic limit $(\tau \gtrsim 1)$ and close to the Fermi surface $\left(+k_{F}\right)$ this function should approach the Green's function of the Luttinger model for right-moving fermions, i. e.

$$
\mathcal{G}_{v_{1}, v_{2}, K_{\rho}}^{(L M)}(k, \tau) \equiv \int d x e^{-i k x} \tilde{\mathcal{G}}_{v_{1}, v_{2}, K_{\rho}}^{(L M)}(x, \tau),
$$

with

$$
\begin{aligned}
& \tilde{\mathcal{G}}_{v_{1}, v_{2}, K_{\rho}}^{(L M)}(x, \tau) \\
& =\frac{e^{i k_{F} x} c}{\sqrt{v_{1} \tau+i x} \sqrt{v_{2} \tau+i x}\left(x^{2}+v_{2}^{2} \tau^{2}\right)^{-\left(K_{\rho}+1 / K_{\rho}-2\right) / 8}},
\end{aligned}
$$

where $c$ is a normalization constant 28 and $k_{F}$ the Fermi momentum. Therefore, in order to identify the spin and charge excitations directly in the Green's function, we carry out a nonlinear $\chi^{2}$ fit of our data for $\mathcal{G}(k, \tau)$ to $\mathcal{G}_{v_{1}, v_{2}, K_{\rho}}^{(L M)}(k, \tau)$. The fit parameters are the two velocities $v_{1}$ and $v_{2}$, and the normalization constant $c 21$ Due to the statistical error in the Quantum-Monte-Carlo data, we get statistical errors $\Delta v_{1}, \Delta v_{2}$, and $\Delta c$ for the parameters $v_{1}, v_{2}$, and $c$, respectively. The splitting of the singleparticle mode into two excitations is thus detected when the difference between the two velocities is larger than the statistical error. Furthermore, in order to make sure that the two excitations coincide with the spin and the charge modes one has to compare $v_{1}$ and $v_{2}$ with the velocities $v_{\rho}$ and $v_{\sigma}$ calculated independently via the susceptibilities.

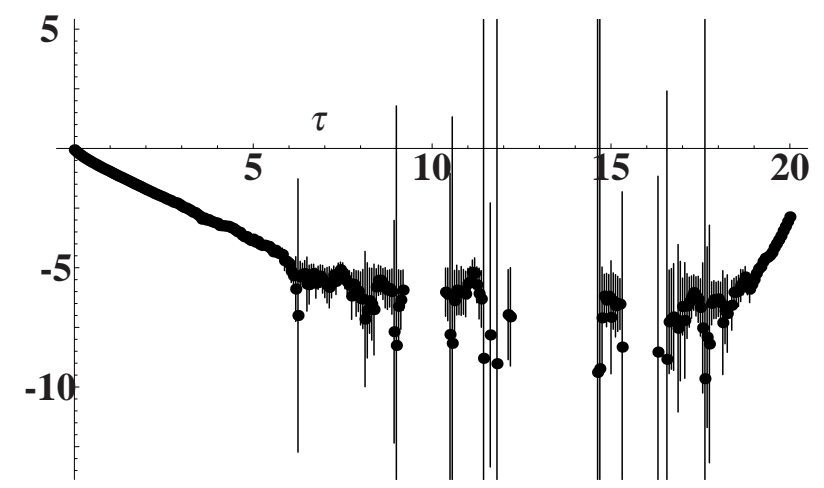

FIG. 5. Logarithmic plot of the imaginary-time Green's function $\mathcal{G}(k, \tau)$ vs $\tau$ with $k=k_{F}+\pi 9 / 64$, as obtained from Quantum-Monte-Carlo data.

However, in order to carry out this fit one should not use the data from the whole interval $0.0 \leq \tau \leq \beta$ for the following reasons. First of all the Hubbard model and the Luttinger model Green's function should coincide only asymptotically. For this reason, we choose $\tau \geq 1.0$. Moreover, the interval $\beta / 2 \leq \tau \leq \beta$ is equivalent to the one $-\beta / 2 \leq \tau \leq 0$ so that we can omit the former. In addition, as can be seen in Fig. 5 the log of the imaginarytime Green's is quite sharply defined up to $\tau \approx 5.0$. For $\tau \gtrsim 5.0$, large (relative) errors start to develop due to the small value of the Green's function in these points. For this reason, we choose to carry out our fit only for the data in the interval $1.0 \leq \tau \leq 5.0$ in order to select the less "noisy" data. In order to check that our results do not depend on this choice, we also carry out the fit for the data in the interval $1.0 \leq \tau \leq \beta / 2=10.0$ (Fig. 7). This turns out to be quite similar to the first one (Fig. 6) except for larger statistical errors. In Fig. 6 we show the result of our fit with the $T=0$ Green's function [ (2) with (3)] for several values of $q=\left(k-k_{F}\right)$. The vertical black lines show the value of the spinon and holon excitation energies $\varepsilon_{1}(q) \equiv v_{1}(q) q$ and $\varepsilon_{2}(q) \equiv v_{2}(q) q$, respectively, obtained from the fit with the single-particle Green's function.29 As one can see, we obtain a clear separation of the two modes for almost all the $q$ points. In addition, the velocities are slightly $q$-dependent as expected from a curved band. To check that these modes correspond to spin and charge degrees of freedom, we plot in the same figure the dispersions calculated from the 
peaks of the corresponding susceptibilities. The width of the gray regions indicate the peak positions within their uncertainty. As one can see, the dispersions obtained in the two ways coincide within the statistical error. We find it remarkable that, even at $k$-points far from $k_{F}$, the fit with the Luttinger-liquid Green's function is in agreement with the two-particle response, although the dispersion is no longer linear. It thus seems that spin and charge separation survives even at higher energies.

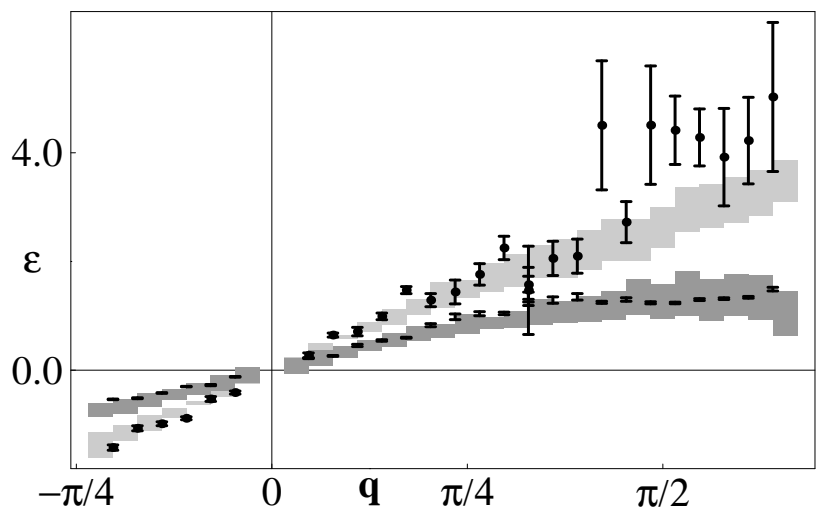

FIG. 6. Spin and charge dispersions $\varepsilon_{1}$ and $\varepsilon_{2}$ vs $q=k-k_{F}$ (errorbars without and with central dot, respectively) as obtained from the $\chi^{2}$ fit of the Quantum-Monte-Carlo data for the imaginary-time Green's function with the Luttinger liquid Green's function [ (2) with (3)]. The Luttinger liquid Green's function is taken at zero temperature and with correlation exponent $K_{\rho}=1$. The fit is carried out for the data in the time interval $1.0 \leq \tau \leq 5.0$. For comparison, we also show the dispersions obtained from the peak positions (with corresponding uncertainty) of the spin (dark gray) and of the charge (light gray) dispersions (Cfr. Figs. 2 and 11).

In Fig. 6 we used the simplest form of (3), namely, the one with $K_{\rho}=1.0$. This is not, in principle, the correct value of the correlation exponent $K_{\rho}$ when $U \neq 0$. Actually, one could use $K_{\rho}$ as a further parameter to fit the data or, alternatively, use the result from the BetheAnsatz solution 30 . It turns out, however, that an attempt to fit $K_{\rho}$ yields an error of the order of 0.5 , which means that $K_{\rho}$ cannot be determined by our fit. It also turns out that the result of the fit does not depend crucially on the value of $K_{\rho}$ we are using. Indeed, as one can see from Fig. 8, where we show the results of the fit obtained with the Bethe-Ansatz value $K_{\rho}=0.7$ the results of the fit do not differ appreciably from the ones in Fig. 6. For this reason, the non-interacting value $K_{\rho}=1.0$ can be safely used. This is important, because in this way it is possible to test the occurrence of spin-charge separation even without knowing whether the system has anomalous scaling $\left(K_{\rho} \neq 1\right)$ or not. This could be useful in cases where the anomalous exponent may be not known a priori and may be difficult to evaluate. In this case, one can

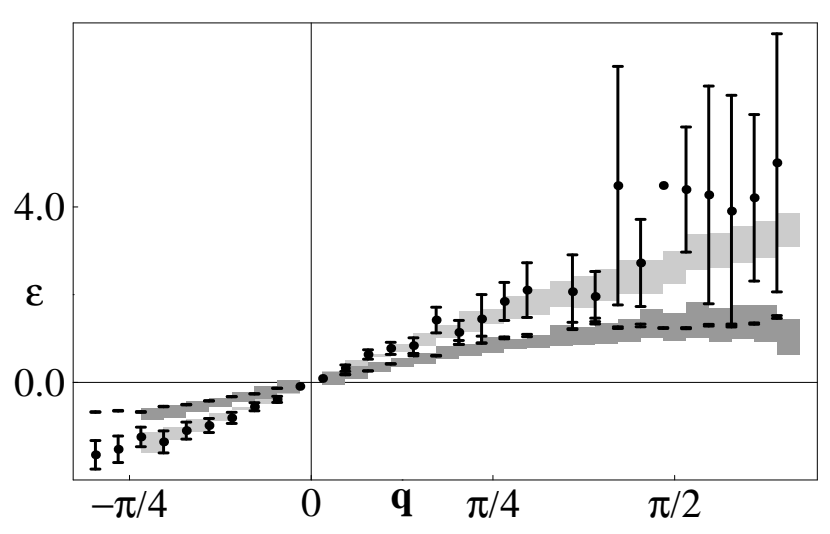

FIG. 7. Same as Fig. 6 except that the fit is carried out for the data in the time interval $1.0 \leq \tau \leq 10.0$.

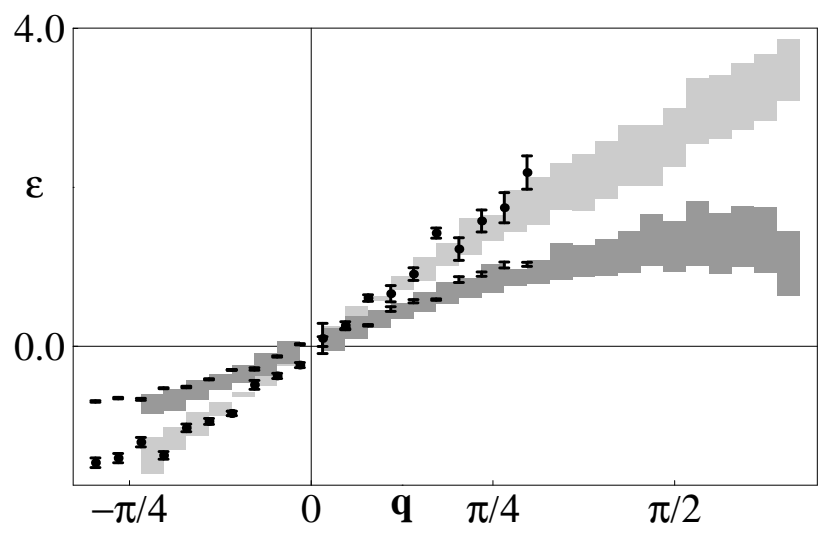

FIG. 8. Same as Fig. 6 except that the fit is carried out with the Luttinger liquid Green's function [ (2) with (3)] with correlation exponent $K_{\rho}=0.7$.

assume a form of the fitting function without spectral anomaly (i. e., $K_{\rho}=1$ ), but simply with a branch cut due to spin-charge separation. This could be useful, for example, to test spin-charge separation in 2-D as we shall discuss below.

Since the Quantum-Monte-Carlo simulations are carried out at finite temperature $\beta=1 /\left(k_{B} T\right)=20$, we also perform our fit with the Luttinger model Green's function at the same temperature $\mathcal{G}_{v_{1}, v_{2} . K_{\rho}}^{(L M)}(k, \tau ; \beta=20)$, which has the same form as (2) with (3) replaced with

$$
\begin{aligned}
& \tilde{\mathcal{G}}_{v_{1}, v_{2}, K_{\rho}}^{(L M)}(x, \tau ; \beta) \\
& =e^{i k_{F} x} \sqrt{g_{v_{1}, 1}\left(\frac{\pi x}{\beta}, \frac{\pi \tau}{\beta} ; \beta\right) g_{v_{2}, K_{\rho}}\left(\frac{\pi x}{\beta}, \frac{\pi \tau}{\beta} ; \beta\right)},
\end{aligned}
$$

with

$g_{v, K}(\tilde{x}, \tilde{\tau} ; \beta)=$ 
$-i\left[\frac{\beta}{\pi}\left(\cosh (\tilde{x})^{2}-\cos (\tilde{\tau})^{2}\right)\right]^{-\frac{K+1 / K}{4}} e^{-i \arg (\tanh \tilde{x}+i \tan \tilde{\tau})}$.

In Fig. 9, we show the fit performed with the more complicated finite-temperature $(\beta=20)$ Green's function (5). As one can see, the results are not appreciably different from the ones of Fig. 6, which means that the temperature of our simulation is low enough and we can safely fit our results with the $T=0$ Green's function.

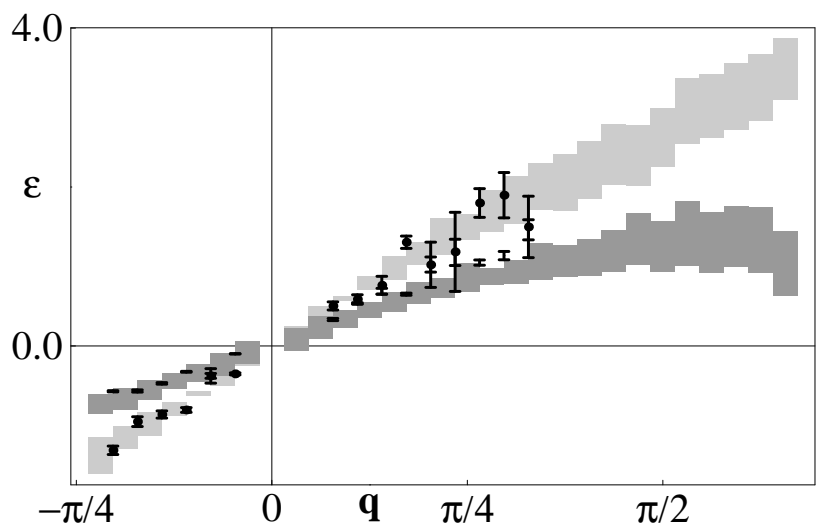

FIG. 9. Same as Fig. 6 except that the fit is carried out with the Luttinger liquid Green's function [ (2) with (5)] with finite temperature $(\beta=20)$ and correlation exponent $K_{\rho}=1$.

Finally, to check that the two different velocities $v_{1}$ and $v_{2}$ obtained are not an artifact of our fit, we carry out a fit of the non-interacting Green's function $\tilde{\mathcal{G}}^{(0)}(k, \tau ; \beta)$ with the function (2) assuming artificially the same statistical errors as the ones obtained in the Quantum-Monte-Carlo simulation. As one can see in Fig. 10, in this case the spin and charge velocities obtained are equal within the statistical error, as it should be.

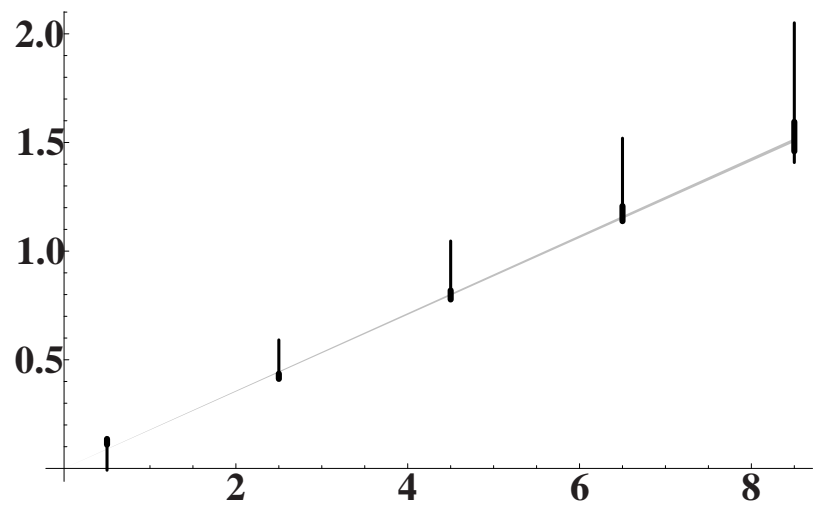

FIG. 10. Fit of the $U=0$ imaginary-time Green's function with Eq. (1). The straight line shows $\omega=v_{F} q$ where $v_{F}$ is the Fermi velocity of the $U=0$ system.
Another motivation of this work was to test a "diagnostic operator" that can be applied to detect numerically the occurrence of spin and charge separation in a manybody system from Quantum-Monte-Carlo data. If one uses exact diagonalization, where the spectral function of the system can be evaluated directly, it is of course not necessary to fit the imaginary-time Green's function. However, we believe that the systems that can be studied by exact diagonalization (10-16 sites) are too small to allow for a systematic study of spin-charge separation (except for a very high value of the momentum, like in Ref. 15). The Fourier transform (in momentum and imaginary-frequency space) of the spin-charge separated Green's function Eq. (3) with $K_{\rho}=1$ reads

$$
\hat{\mathcal{G}}_{v_{1}, v_{2}, K_{\rho}}^{(L M)}(k, \omega) \propto \frac{1}{\sqrt{i \omega-\varepsilon_{1}(k)} \sqrt{i \omega-\varepsilon_{2}(k)}},
$$

where $\varepsilon_{1}(k)=v_{1}\left(k-k_{F}\right)$ and $\varepsilon_{2}(k)=v_{2}\left(k-k_{F}\right)$ represent the spin and charge excitations (measured from the chemical potential) in which the single-particle excitation is split. The same form of $\hat{\mathcal{G}}_{v_{1}, v_{2}, K_{\rho}}^{(L M)}(k, \omega)$ could be expected to hold asymptotically, i.e. for small frequencies and close to the Fermi surface, in higher dimensions. Close to the Fermi surface, one will have a directiondependent dispersion $\varepsilon_{i}(\boldsymbol{k})=\left(\boldsymbol{k}-\boldsymbol{k}_{F}\right) \cdot \boldsymbol{v}\left(\boldsymbol{k}_{F}\right)$ where $v\left(\boldsymbol{k}_{F}\right)$ is the Fermi velocity of the point at the Fermi surface $\boldsymbol{k}_{F}$ closest to $\boldsymbol{k}$. Spin-charge separation would be signaled by two different, direction-dependent $\varepsilon_{1}(\boldsymbol{k})$ and $\varepsilon_{2}(\boldsymbol{k})$ for a given $\boldsymbol{k}$.

\section{CONCLUSIONS}

To summarize, we carried out a test of spin-charge separation in the 1-D Hubbard model at finite doping. It is in general difficult to resolve the peaks corresponding to the spin and charge excitations in the single-particle spectral function due to the loss of accuracy which occurs when analytically continuing the imaginary-time Quantum-Monte-Carlo results to real frequencies. For some values of the momentum close to the Fermi surface, however, we were able to resolve two peaks whose energies correspond to the peaks at the same $q=k-k_{F}$ in the spin and charge susceptibilities, respectively.

By fitting the Quantum-Monte-Carlo data for the imaginary-time Green's function with the exact solution from the Luttinger model with the spin and charge velocities as fitting parameters, we have been able to resolve the two excitations over the whole Brillouin zone. The two excitation energies found in the fit agree, within statistical error, with the spin and charge excitations, respectively, identified with the peaks of the spin and charge susceptibilities. Remarkably, this occurs also away from the region where the band dispersion is linear. We also suggested a possible extension of this "diagnostic operator" to test a possible occurrence of spin-charge separation in two dimensions. 


\section{ACKNOWLEDGMENTS}

We thank J. Voit, W. von der Linden, F. Mila, and M. Imada for stimulating discussions and precious suggestions. This work was partially supported by the Bavarian high-Tc program FORSUPRA and by the BMBF (05 605 WWA 6). M. G. Z. acknowledges support from the DFN project Tk598-VA/D3. E. A. acknowledges research support from the TMR program ERBFMBICT950048 of the European Community. J. R. S. acknowledges support from NSF grant DMR-9629987. The numerical calculations were carried out at the HLRZ Jülich.

${ }^{1}$ V. J. Emery, in Highly-Conducting One-Dimensional Solids, edited by J. T. Devreese, R. E. Evrard, and V. van Doren (Plenum Press, New York, 1979).

${ }^{2}$ F. D. M. Haldane, J. Phys. C 14, 2585 (1981).

${ }^{3}$ J. Voit, Reports on Progress in Physics 58, 977 (1995).

${ }^{4}$ E. H. Lieb and F. Y. Wu, Phys. Rev. Lett. 20, 1445 (1968).

${ }^{5}$ V. Meden and K. Schönhammer, Phys. Rev. B 46, 15753 (1992).

6 J. Voit, J. Phys. Cond. Matt. 5, 8305 (1993).

${ }^{7}$ P. W. Anderson, Phys. Rev. B 42, 2624 (1990).

${ }^{8}$ P. W. Anderson, Phys. Rev. Lett. 64, 1839 (1990).

${ }^{9}$ P. W. Anderson, Science 258, 672 (1992).

${ }^{10}$ D. Clarke, S. Strong, and P. Anderson, Phys. Rev. Lett. 74, 4499 (1995).

${ }^{11}$ P. W. Anderson, Phys. Rev. B 55, 11785 (1997).

12 D. G. Clarke, S. P. Strong, and P. W. Anderson, Phys. Rev. Lett. 72, 3218 (1994).

${ }^{13}$ K. Penc et al., Phys. Rev. Lett. 77, 1390 (1996).

14 M. Ogata and H. Shiba, Phys. Rev. B 41, 2326 (1990).

${ }^{15}$ E. A. Jagla, K. Hallberg, and C. A. Balseiro, Phys. Rev. B 47, 5849 (1993).

${ }^{16}$ W. Putikka et al., Phys. Rev. Lett. 73, 170 (1994).

${ }^{17}$ R. Eder and Y. Ohta, Phys. Rev. B 56, 2542 (1997).

18 T. Tohyama and S. Maekawa, J. Phys. Soc. Japan 65, 1902 (1996).

19 C. Kim et al., Phys. Rev. Lett. 77, 4054 (1996).

${ }^{20}$ R. Preuss, A. Muramatsu, W. von der Linden, P. Dieterich, F. F. Assaad, W. Hanke, Phys. Rev. Lett. 73, 732 (1994).

${ }^{21}$ The results of the fit turns out to depend very weakly on the value of $K_{\rho}$ used (cf. Sec. III).

${ }^{22}$ J. E. Hirsch, Phys. Rev. B 28, 4059 (1983).

${ }^{23}$ S. R. White et al., Phys. Rev. B 40, 506 (1989).

${ }^{24}$ R. N. Silver et al., Phys. Rev. Lett. 65, 496 (1990).

${ }^{25}$ J. E. Gubernatis et al., Phys. Rev. B 44, 6011 (1991).

${ }^{26}$ H. J. Schulz, Int. J. Mod. Phys. B 5, 57 (1991).

${ }^{27}$ D. Pines and P. Nozières, The Theory of Quantum Liquids (W. A. Benjamin, inc., New York, 1966), Vol. I.

${ }^{28}$ It is not possible to fix the constant $c$ through sum rules, since the two Green's functions coincide only asymptotically.

${ }^{29}$ The $q$-dependence of the velocities is due to the fact that we fit independently $\mathcal{G}\left(k_{F}+q, \tau\right)$ for each $q$.

${ }^{30}$ H. J. Schulz, Int. J. Mod. Phys. 5, 57 (1991). 